\title{
Study of Hypoglycemia in Neonates with Low Birth Weight
}

\author{
Abrar Ahmed Siddique ${ }^{1}$, N.L Sridhar ${ }^{2}$ \\ ${ }^{1}$ Paediatrics, Assistant Professor, Niloufer Hospital, Hyderabad, ${ }^{2}$ Professor, Kamineni Institute of Medical Sciences, Hyderabad.
}

\section{Abstract}

Background: Glucose metabolism disorders are common in low birth weight (LBW) infants and are associated with high morbidity and mortality. Neonatal hypoglycemia, a common metabolic problem, often goes unnoticed owing to lack of specific symptoms. Hypoglycemia both symptomatic and asymptomatic can lead to long term neurological sequelae. Therefore, it needs early management to prevent brain damage in a developing neonate. Subjects and Methods: This study was conducted to evaluate the prevalence and risk factors associated with hypoglycemia in low birth weight infants. Design: A hospital-based prospective longitudinal study. Duration: One year (October $2017-$ October 2018). Setting: Niloufer Hospital, Hyderabad. Participants: 50 LBW neonates with birth weight less than 2500 grams. Methods: Blood glucose values were measured at the age of $1 \mathrm{~h}, 6 \mathrm{~h}, 12 \mathrm{~h}, 24 \mathrm{~h}$ and $48 \mathrm{~h}$ after delivery which was independent of feeding time. Blood glucose value less than $40 \mathrm{mg} / \mathrm{dl}(2.2 \mathrm{mmol} / \mathrm{l})$ was defined as hypoglycemia. For statistical analysis, SPSS software version 20 was used. Result: Out of 50 neonates, $15(30 \%)$ had one or more episode of hypoglycemia. Overall 22 episodes were recorded. Out of 15 hypoglycemic neonates $8(53.3 \%)$ were small for gestational age (SGA) and 7(46.7\%) were AGA. Sepsis was significantly noticed after hypoglycemia. The pattern of blood glucose levels was significantly different among hypoglycemic babies and normoglycemic babies over first 72 hours. Conclusion: Hypoglycemia was frequent among low birth weight babies more so in SGA babies in first 24 hours.

Keywords: Hypoglycemia, Low birth weight newborns, Sepsis, Hypoglycemic episode.

Corresponding Author: Dr. N.L Sridhar, Professor, Kamineni Institute of Medical Sciences, Hyderabad.

Email: drnlsridhar4@gmail.com

Received: March 2020

Accepted: March 2020

\section{Introduction}

Low birth weight (LBW) has been defined by WHO as weight at birth of less than $2.5 \mathrm{~kg}$. By international agreement, LBW has been defined as a birth weight of less than 2500 grams, with the measurement being taken preferably within the first hour of life, before significant postnatal weight loss has occurred. ${ }^{[1]}$ It contributes substantially to neonatal, infant, and childhood mortality and morbidity. Across the world, neonatal mortality is 20 times more likely for LBW babies compared to NBW babies $(>2.5 \mathrm{~kg}) \cdot{ }^{[2]}$ It is now a well-recognized fact that birth weight is not only a critical determinant of child survival, growth, and development, but also a valuable indicator of maternal health, nutrition, and quality of life. Neonatal hypoglycemia is a common metabolic disease due to inability to maintain glucose homeostasis. ${ }^{[3]}$ The overall prevalence depends on birth weight, gestational age and intrauterine growth retardation. Since quite a proportion of neonates do not manifest any symptoms despite existence of hypoglycemia, exact incidence is difficult to work out. In other words, symptomatic hypoglycemia is not common as against chemical hypoglycemia. Undiagnosed hypoglycemia can have long term neurological consequences; thus, the emphasis is on prevention and early detection along with treatment of asymptomatic hypoglycemia. ${ }^{[4]}$ Prognosis of these newborns with hypoglycemia depends on how promptly and efficiently hypoglycemia is managed. ${ }^{[5,6]}$ The maintenance of normoglycemia in newborns depends upon adequacy of glycogen stores, maturation of glycogenolytic and gluconeogenic pathways and an integrated endocrine response. We therefore propose to study clinical profile of low birth weight babies with reference to occurrence of hypoglycemia.

\section{Subjects and Methods}

Place of Study: Niloufer Hospital, Hyderabad.

Type of Study: A hospital-based prospective longitudinal study.

Sample Collection: Capillary blood was collected by heel prick after proper aseptic measures and also venous blood was collected.

\section{Sampling Methods:}

Capillary blood was screened by reagent strips method and the same time venous blood sample was sent for laboratory confirmation by glucose oxidase method in an autoanalyzer.

\section{Inclusion Criteria:}

Neonates with birth weight less than 2500 grams. 
Exclusion Criteria: Infants of diabetic mother, those with birth asphyxia, congenital malformations and endocrine deficiencies were excluded.

\section{Statistical Methods:}

Data were presented in the form of statistical Tables and charts. SPSS software version 20 was used.

\section{Results}

The age of mothers ranged from 23 years to 35 years. Mean with SD age of mothers was $26.9 \pm 3.9$ years. Total 17 neonates had maternal morbidities out of which eight had leaking per vagina. Statistically no significant maternal morbidity was associated with occurrence of hypoglycemia. Apgar score at $1 \mathrm{~min}$ ranged from 2 to 7 with mean of $7 \pm 1$ and at 5 min ranged from 8 to 9 with mean of $9 \pm 1$. Out of 50,35 neonates $(70 \%)$ were normoglycemic and 15 neonates $(30 \%)$ had hypoglycemic episodes. Overall 22 episodes of hypoglycemia were recorded. There was no significant association between birth weight and episodes of hypoglycemia. Majority of hypoglycemic infants were male. There was no significant association between gender and episodes of hypoglycemia.

Majority of hypoglycemic infants were born with low birth weight (1.501-2.500 kg). There was no significant association between birth weight and episodes of hypoglycemia.

Table 1: Comparison of hypoglycemic and normoglycemic neonates with respect to birth weight

\begin{tabular}{|l|l|l|l|l|l|}
\hline Birth Weight $(\mathbf{K g})$ & $\mathbf{N}$ & Infants & $\mathbf{\%}$ & Normoglycemic(n=35) & P Value \\
\cline { 3 - 7 } & & Hypoglycemic(n=15) & 6.6 & 1 & $* 0.203$ \\
\hline Extremely Low $(\leq 1.000 \mathrm{~kg})$ & 2 & 1 & 26.6 & 6 & 2.8 \\
\hline Very Low $(1.001-1.500 \mathrm{Kg})$ & 10 & 4 & 66.6 & 28 & 17.1 \\
\hline Low(1.501-2.500Kg) & 38 & 10 & & 80.0 \\
\hline
\end{tabular}

Table 2: Comparison of hypoglycemic and normoglycemic neonates with respect to gestational size

\begin{tabular}{|l|l|l|l|l|l|l|}
\hline Gestational size & $\mathbf{N}$ & Infants & P Value \\
\cline { 3 - 7 } & & Hypoglycemic(n=15) & \% & Normoglycemic(n=35) & \\
\hline AGA & 34 & 7 & $20.5 \%$ & 27 & $79.5 \%$ \\
\hline SGA & 16 & 8 & $50 \%$ & 8 & $50 \%$ & 0.20 \\
\hline
\end{tabular}

AGA - Appropriate for Gestational Age

SGA- Small for Gestational Age

Out of fifteen hypoglycemic infants eight were small for gestational age and seven were appropriate for gestational age. $50 \%$ of all the small for gestational age neonates were hypoglycemic and $20.5 \%$ of all the appropriate for gestational age were hypoglycemic. This was statistically significant $(\mathrm{p}$ value $=0.020)$. Hypoglycemia was more common in small for gestational age infants.

Various co-morbidities which occurred after hypoglycemia were analysed in normoglycemics and hypoglycemic group. Significant difference was analysed with sepsis occurring in hypoglycemic infants.

\section{Discussion}

The term "hypoglycemia" refers to a low blood glucose concentration. Neonatal hypoglycemia is not a medical condition in itself, but a feature of illness or of failure to adapt from the fetal state of continuous transplacental glucose consumption to the extrauterine pattern of intermittent nutrient supply. There is controversy over the definition of a "safe" blood glucose concentration, that is, a value below which there is risk of long-term neurodevelopmental impairment. Hypoglycemia associated with abnormal clinical signs (symptomatic hypoglycemia) has a poor short- and long-term outcome but evidence of risk in the absence of clinical signs (asymptomatic hypoglycemia) is inconclusive. Hypoglycemia is not a disease but a symptom of other diseases or lack of metabolic adaptation postnatally. Manifestations of hypoglycemia are non-specific but unexplained by other diagnoses and corrected with the provision of glucose. ${ }^{[7]}$ However, when the low blood glucose levels are prolonged or recurrent, they may result in acute systemic effects and neurologic sequelae. According to AAP guideline 2011, there has been no substantial evidence-based progress in defining what constitutes clinically important [neonatal hypoglycemia $(\mathrm{NH})$, particularly regarding how it relates to brain injury, and that monitoring for, preventing and treating $\mathrm{NH}$ remain largely empirical. The guidelines call for immediate intravenous glucose for infant who are symptomatic and have glucose levels lower than $40 \mathrm{mg} / \mathrm{dl}$. For asymptomatic at-risk infants, the initial feed should be given within $1 \mathrm{~h}$ of birth, with glucose screening $30 \mathrm{~min}$ after the first feed. Because there is no point-of-care screening method reliable enough to be used as the sole method for screening for $\mathrm{NH}$, the blood or plasma glucose concentration must be confirmed by laboratory testing done simultaneously. In our study too, besides doing the reagent strip test, blood was sent for laboratory measurement of glucose immediately. However, therapy required was initiated based on reagent strip test itself. The incidence of hypoglycemia in low birth weight neonates in our study was $30 \%$. Different studies in literature have reported varying incidences. Dias E and Gada $\mathrm{S},{ }^{[8]}$ reported the incidence of hypoglycemia (Blood glucose 
$<40 \mathrm{mg} / \mathrm{dl}$ ) to be $17 \%$ whereas Jonas D et al, ${ }^{[9]}$ reported $11.7 \%$ incidence of hypoglycemia. Yoon JY et al, ${ }^{[10]}$ reported $20 \%$ incidence in which hypoglycemia was defined as blood glucose level of less than $40 \mathrm{mg} / \mathrm{dl}$ up to 24 hours and less than $50 \mathrm{mg} / \mathrm{dl}$ thereafter. Dashti $\mathrm{N}$ et al, ${ }^{[11]}$ reported hypoglycemia incidence to be $15.15 \%$ which was less as compared to our study. Our study demonstrated significantly greater incidence of hypoglycemia in LBW newborns. The mean blood glucose values in the LBW newborns were significantly lower at $1 \mathrm{~h}, 6 \mathrm{~h}, 12 \mathrm{~h}, 24 \mathrm{~h}$ and $48 \mathrm{~h}$ after delivery, which is well correlated with the findings of Singhal et al. ${ }^{[12]}$ and Anderson et al. ${ }^{[13]}$ In the present study, significantly lower mean blood glucose values and higher incidence of hypoglycemia was found in SGA than the AGA babies [Table 2]. Holtrop $\mathrm{PC},{ }^{[14]}$ studied the incidence of hypoglycemia in the SGA babies. Definition of hypoglycemia chosen was that suggested by Srinivasan et al. ${ }^{[15]}$ Hypoglycemia was detected in $14.7 \%$ of SGA babies which was much lower than that of the present study. Higher incidence in the present study may be due to large number of the LBW infants present in the SGA group which is also a contributing factor of hypoglycemia.Bhat et al. ${ }^{[16]}$ found hypoglycemia in $25.2 \%$ of SGA babies as compared to $32.8 \%$ reported by Lubchenco and Bard. ${ }^{[17]}$ The variability in incidence could be partly due to different definitions used for hypoglycemia, SGA and different policies of feeding. Various co-morbidities were analysed in normoglycemic and hypoglycemic infants of which sepsis was significantly noticed after hypoglycemia.

\section{Conclusion}

According to our observation, the incidence of hypoglycemia in low birth weight neonates in our study is $24 \%$. Small for gestational age is a significant determinant for hypoglycemia. Hypoglycemic episodes were significantly noticed in first 24 hours as compared to other time interval. Various co-morbidities were analysed in normoglycemic and hypoglycemic infants of which sepsis was significantly noticed after hypoglycemia.

\section{References}

1. World Health Organization. Hypoglycemia of the newborn: Review of the Literature. Geneva: WHO/CHD/97.1;1997. p. 1-55.

2. Ktorza A, Bihoreau MT, Nurjhan N, et al. Insulin and glucagon during the perinatal period: secretion and metabolic effects on the liver. Biol Neonate. 1985;48(4):204-20. DOI:10.1159/000242173

3. Adamkin DH. Postnatal Glucose Homeostasis in Late preterm and term infants. A clinical report from American Academy of Pediatrics, Committee on Fetus and NewbornPediatrics. Pediatrics 2011;127:5759 .

4. Barbara J. Stoll. The Endocrine System. Nelson Textbook of Pediatrics 18 th ed., vol. 1. Part XI, Chapter 107. Mosby, Churchill Livingstone: Elsevier Saunders; 2007. p. 782-5.

5. Hawdon JM, Ward Platt MP, Aynsley-Green A. Patterns of metabolic adaptation for preterm and term infants in the first neonatal week. Arch Dis Child. 1992 Apr;67(4 Spec No):357-65.

6. Hewitt V, Watts R, Robertson $\mathrm{J}$, et al. Nursing and midwifery management of hypoglycaemia in healthy term neonates. Int J Evid Based Healthc. 2005 Aug;3(7):169-205. doi: 10.1111/j.14796988.2005.00025.x.

7. Hoseth E, Joergensen A, Ebbesen F, et al. Blood glucose levels in a population of healthy, breast fed, term infants of appropriate size for gestational age. Arch Dis Child Fetal Neonatal Ed. 2000 Sep;83(2):F117-9. DOI:10.1136/fn.83.2.f117

8. Dias E, Gada S. Glucose levels in newborns with special reference to hypoglycemia: a study from rural India. J ClinNeonatol. 2014 Jan;3(1):35-8. doi: 10.4103/2249-4847.128729.

9. Jonas D, Dietz W, Simma B. Hypoglycemia in newborn infants at risk. KlinPadiatr. 2014 Sep;226(5):287-91. doi: 10.1055/s-0034-1385928. Epub 2014 Aug 25.

10. Yoon JY, Chung HR, Choi CW, et al. Blood glucose levels within 7 days after birth in preterm infants according to gestational age. Ann PediatrEndocrinolMetab. 2015 Dec;20(4):213-9. doi 10.6065/apem.2015.20.4.213. Epub 2015 Dec 31.

11. Dashti N, Einollahi N, Abbasi S. Neonatal hypoglycemia: prevalence and clinical manifestations in Tehran children's hospital. Pakistan J Med Sci Online. 2007;23(3):340-3.

12. Singhal PK, Singh M, Paul VK. Prevention of hypoglycemia. A controlled evaluation of sugar fortified feeding in small for date infants. Indian Pediatr1992;29:1365-9.

13. Anderson S, Shakya KN, Shrestha LN, de L Costello AM. Hypoglycemia: A common problem among uncomplicated newborn infants in Nepal. J Trop Pediatr1993;39:273-7.

14. Holtrop PC. The frequency of hypoglycemia in full-term large and small for gestational age newborns. Am J Perinatol. 1993 Mar;10(2):150-4

15. Srinivasan G, Pildes RS, Cattamanchi G, et al. Plasma glucose values in normal neonates: a new look. J Pediatr. 1986 Jul;109(1):114-7.

16. Bhat MA, Kumar P, Bhansali A, et al. Hypoglycemia in small for gestational age babies. Indian J Pediatr. 2000 Jun;67(6):423-7.

17. Lubchenco LO, Bard $\mathrm{H}$. Incidence of hypoglycemia in newborn infants classified by birth weight and gestational age. Pediatrics. 1971 May;47(5):831-8.

Copyright: (C) the author(s), 2020. It is an open-access article distributed under the terms of the Creative Commons Attribution License (CC BY 4.0), which permits authors to retain ownership of the copyright for their content, and allow anyone to download, reuse, reprint, modify, distribute and/or copy the content as long as the original authors and source are cited.

How to cite this article: Siddique AA, Sridhar NL. Study of Hypoglycemia in Neonates with Low Birth Weight. Asian J.Clin.Pediatr.Neonatol.2020;8(1):44-46.

DOI: dx.doi.org/10.47009/ajcpn.2020.8.1.11

Source of Support: Nil, Conflict of Interest: None declared. 